\title{
EVALUACIÓN DE UN ARCHIVO DE HISTORIAS CLÍNICAS MEDIANTE LA APLICACIÓN DE MÉTODOS DE CONTROL ESTADÍSTICO DE LA CALIDAD
}

\author{
Paula Asensio Villahoz (1) y Juan Antonio Vicente Vírseda (2)
}

(1) Unidad de Codificación Clínica. Hospital Universitario "Río Hortega". Valladolid.

(2) Facultad de Ciencias Económicas y Empresariales. UNED. Madrid.

RESUMEN
Fundamentos: La cumplimentación del Conjunto Mínimo Básico de
Datos (CMBD) de hospitalización es obligatoria para todos los hospita-
les. Depende de un buen funcionamiento del Archivo de Historias Clíni-
cas. El objetivo de este estudio es hacer una evaluación continua de la ca-
lidad del Archivo de Historias Clínicas con técnicas estadísticas de
Diagramas de Control.
Métodos: De junio2005 a enero2009 se realizó una búsqueda retros-
pectiva de las altas pendientes de codificar en el CMBD de un hospital de
tercer nivel. Se registraron en Excel las altas/Historias Clínicas: buscadas,
encontradas, prestadas, con incidencias y no localizadas de cada mes/año
estudiado. Se calculó el Índice de Eficacia Global, considerándose el va-
lor del estándar de calidad 0,9. Y para conocer la calidad del proceso a lo
largo de todo el período de estudio y en cada mes/año analizado, se apli-
caron los test gráficos del Diagrama de Control de Shewhart y de Sumas
Acumuladas (CUSUM).
Resultados: El Índice de Eficacia Global fue 0,95 . Los mayores \% de
errores correspondieron a noviembre2008 con 55 (11,73\%) y junio2008
con 14 (10,45\%). En los Diagramas de Control destacan los valores anor-
malmente altos, obtenidos tras la estandarización, en julio2005 (7,42) y
noviembre2008 (7,00). Y en CUSUM se observó un punto fuera de con-
trol al inicio, julio2005 (5,95), aunque hasta junio2007 la media (4,70\%)
se situó ligeramente por debajo de la media global (4,81\%). Aumentando
a partir de julio2007 (5,02\%).
Conclusión: La calidad del Archivo ha ido variando a lo largo del tiempo, siendo más baja especialmente al final del estudio.

Palabras clave: Sistemas de Información. Codificación Clínica. Archivos de Historias Clínicas. Control de Calidad.

\section{ABSTRACT}

\section{Evaluation of A Medical Records \\ Archive through the Application of Statistical Methods for Quality Control}

Background: In undertaking a Minimum Basic Data Set (MBDS) of hospital discharges has to be done for all hospitals. It depends on the good function of the Medical Records Archive. The objective of this study is the continuous evaluation of the quality of the Medical Records Archive via Control Diagrams statistical techniques arose.

Methods: From June2005 to January2009 a retrospective search of the discharges/Medical Records pending coding, from a tertiary care university hospital was carried out. The different categories of discharges/Medical Records were registered on an Excel spreadsheet under the following headings: searched for, found, completed/closed, pending and not located for each a particular month and year. The Overall Effectiveness Index was calculated, 0,9 being the standard quality value. Furthermore to assess the process quality over the entire length of the period studied and in each month and year, the Shewhart's Control Diagram and Cumulative Sum (CUSUM) graphic tests were applied.

Results: The Overall Effectiveness Index was 0.95 . The majority of \% errors corresponded to November2008 with $55(11.73 \%)$ and June2008 with $14(10.45 \%)$. In the Control Diagrams there are abnormally high values, obtained after standardization, in July2005 (5.95) and November 2008 (7.00). The CUSUM showed one point outside of the initial control (July2005-5.95), although until June2007, the average (4.7\%) was slightly below the overall averages $(4.81 \%)$ and then increases from July 2007 on $(5.02 \%)$.

Conclusion: The Archive quality has varied over time, demonstrating that it was especially lower at the end of the study.

Key words: Information Systems. Clinical Coding, Medical Records Department. Quality Control.

Paula Asensio Villahoz

Unidad de Codificación Clínica

Hospital Universitario "Río Hortega"

C/ Dulzaina, 2

47012. Valladolid

pasensiovi@saludcastillayleon.es 


\section{INTRODUCCIÓN}

El establecimiento del sistema de información clínica, basado en la extracción de un mínimo número de datos básicos de cada alta hospitalaria es lo que constituye el Conjunto Mínimo Básico de Datos (CMBD) de hospitalización. El registro del CMBD, tanto de hospitalización como de los procedimientos ambulatorios especializados, es de obligada cumplimentación para todos los hospitales del Sistema Nacional de Salud, en nuestro caso particular desde $1992^{1}$. Este registro incluye diversos tipos de datos, siendo los de carácter clínico, es decir, diagnósticos y procedimientos realizados, los que se deben extraer para su codificación de la documentación clínica generada en el proceso asistencial finalizado y debe ser remitida al Archivo de Historias Clínicas. Una vez cumplimentado el CMBD se debe remitir trimestralmente a la correspondiente administración sanitaria $y$, de forma acumulativa, anualmente ${ }^{2,3}$.

Sin entrar en el tema de la calidad de la codificación, que sería motivo de otro trabajo, esto supone que el personal codificador o la Unidad de Codificación Clínica debe acceder al $100 \%$ de las altas hospitalarias. Para ello es clave no sólo el adecuado trabajo de los profesionales clínicos que intervienen en el proceso asistencial como autores de la información clínica que debe figurar en la correspondiente documentación clínica, sino también de los circuitos de dicha documentación para su almacenamiento y custodia. Tales tareas son responsabilidad del Archivo de Historias Clínicas ${ }^{4-6}$, por lo que el correcto funcionamiento del mismo es fundamental, además de para las otras funciones de la documentación e información clínica, para la cumplimentación del $\mathrm{CMBD}^{1-3}$ por parte de una Unidad de Codificación Clínica.

La necesidad de desarrollar e implantar programas de control de calidad en los cen- tros asistenciales y, más concretamente, en los diversos servicios o áreas clínicas, es indiscutible. Existen varios modelos de gestión de la calidad (Joint Commission ${ }^{7}$, $\mathrm{ISO}^{8}, \mathrm{EFQM}^{9}$ ) siendo preferentemente elegido uno u otro generalmente según las características de los diversos servicios o áreas clínicas a evaluar. En el caso concreto del Archivo de HC si bien son escasos los trabajos sobre el desarrollo de programas de control de calidad de los Archivos de Historias Clínicas ${ }^{10}$ sí están descritos varios indicadores de calidad ${ }^{4-6,11}$.

La implantación de sistemas de control de calidad en el Archivo de Historias Clínicas es una necesidad intrínsecamente derivada del papel que éste tiene asignado dentro de las instituciones sanitarias ${ }^{4-6,10-11}$. El indicador de calidad clave y primario es la disponibilidad, en tiempo y en lugar, de la documentación clínica o $\mathrm{HC}^{4-5,10-11}$, indicador común para cualquier soporte y/o formato de la Historia Clínica ${ }^{12,13}$. Si bien, teniendo en cuenta la ineficiente búsqueda bibliográfica, el control de calidad o la implantación de programas de control de calidad desde una perspectiva longitudinal, es decir, viendo cómo cambia la calidad en los Archivos de Historias Clínicas a lo largo del tiempo, es un tema poco estudiado. Sí existen múltiples estudios de tipo transversal en base al cálculo de diversos indicadores de calidad definidos para estos servicios o unidades $^{4-6,10-11}$. Igualmente existen muchos trabajos sobre la calidad de la información clínica recogida en la $\mathrm{HC}$ y/o sobre los diversos formatos de la $\mathrm{HC}$ o soportes de dicha información. Estos últimos temas, muy actuales ante la incorporación de las nuevas tecnologías de la información (TIC's) dentro de la asistencia sanitaria, conllevan la transformación de los tradicionales Archivos de Historias Clínicas en papel en Archivos de HC electrónicas, previo paso generalmente por la digitalización de la $\mathrm{HC}^{12-14}$.

Los Diagramas de Control y, en concreto, 
el método de suma acumulada, es una técnica de control estadístico de la calidad utilizada originariamente en la industria ${ }^{15,16}$. Se trata de una técnica estadística de análisis secuencial de datos con la que se puede ver, de forma rápida y gráficamente, cuánto se aparta una unidad de producto de un estándar previamente establecido, determinando el valor aceptable o inaceptable de tal desviación. Estos gráficos de control permiten monitorizar en forma continua las diferencias acumuladas de una característica cualitativa de un proceso determinado, que puede ser traducida en datos recogidos secuencialmente, permitiendo la detección precoz de desviaciones de un estándar establecido ${ }^{16-18}$. Las ventajas de estas pruebas secuenciales cuando se comparan con los métodos tradicionales de análisis de resultados son la independencia del tamaño muestral, una mayor potencia para detectar cambios transitorios en las tendencias, la continuidad del análisis en el tiempo y la posibilidad de realizar una evaluación rápida de los datos y una identificación rápida de las tendencias ${ }^{19}$.

Los problemas o dificultades de acceso a la documentación clínica versus información clínica para la cumplimentación del estándar: codificación del $99 \%$ de las altas hospitalarias del año, establecido para el CMBD anual por las correspondientes autoridades sanitarias como objetivo del hospital, hizo que nos planteáramos la evaluación de la calidad del Archivo de Historias Clínicas a través de técnicas estadísticas de Diagramas de Control como los de Shewhart y el Diagrama o Método de Suma Acumulada (CUSUM), como una forma de evaluación continua y externa de la calidad del Archivo de Historias Clínicas, puesto que el personal de ambas Unidades, Archivo de Historias Clínicas y Unidad de Codificación Clínica, son independientes o autónomas, aunque dentro del organigrama del hospital pertenezcan al mismo servicio, el Servicio de Admisión y Documentación Clínica.
El objetivo de este estudio es hacer una evaluación continua de la calidad del Archivo de Historias Clínicas con técnicas estadísticas de Diagramas de Control.

\section{MATERIAL Y MÉTODOS}

Durante el período comprendido entre junio de 2005 y enero del 2009 se realizó una búsqueda retrospectiva de las altas hospitalarias pendientes de codificar en el registro del CMBD de hospitalización de un hospital de tercer nivel, finalizado el trimestre en cuestión y hasta el cierre anual del mismo ${ }^{4}$, indistintamente de su estado en el registro, de su ubicación o localización en el momento de la búsqueda que había en el archivo . Se anotaron cada día/mes/año las circunstancias $\mathrm{y} / \mathrm{o}$ problemas detectados cara a su acceso para su codificación en el plazo previamente establecido para la cumplimentación del CMBD y su envío a las correspondientes administraciones sanitarias. Esto suponía revisar una misma Historia Clínica varias veces y/o días, considerándose como oportunidades nuevas cada búsqueda, la cual finalizaba, como ya se ha dicho anteriormente, con la correspondiente codificación del alta hospitalaria.

Se realizó un registro continuo en una hoja de cálculo de Microsoft Excel de los resultados obtenidos sobre las historias clínicas/altas (HC) según los epígrafes: HC buscadas, $\mathrm{HC}$ encontradas, $\mathrm{HC}$ prestadas (altas mal gestionadas informáticamente), $\mathrm{HC}$ con incidencias (sobres vacíos, incompletos...) y $\mathrm{HC}$ que no se localizaron (pérdidas), de las múltiples muestras realizadas, correspondiendo cada muestra a un período mes/año concreto. Se realizó un análisis retrospectivo con el cálculo del Índice de Eficacia Global en primer lugar, considerándose el valor de 0,9 como límite inferior indicativo de control de calidad del Archivo de Historias Clínicas, siendo lo ideal el valor 1 , es decir, el número $\mathrm{HC}$ buscadas es igual al número $\mathrm{HC}$ encontradas. 
En segundo lugar, para conocer la calidad del proceso a lo largo de todo el período de estudio y en cada una de las muestras analizadas, se diseñó un test gráfico en el que la hipótesis nula $\left(\mathrm{H}_{0}\right)$ fue que el proceso estaba bajo control y la hipótesis alternativa $\left(\mathrm{H}_{1}\right)$ que no lo estaba, denominado Diagrama de Control de Shewhart. La construcción del diagrama se realizó representando la característica a medir, w, y las líneas siguientes:

$$
\begin{array}{ll}
\mathrm{LSC}=\mu_{\mathrm{v}}+\mathrm{k}^{*} \sigma_{\mathrm{w}} & \text { (Límite Superior de Control) } \\
\mathrm{LC}=\mu_{\mathrm{v}} \quad \text { (Línea Central } \circ \text { de proceso) } \\
\mathrm{LIC}=\mu_{\mathrm{w}}-\mathrm{k}^{*} \sigma_{\mathrm{w}} \quad \text { (Límite Inferior de Control) }
\end{array}
$$

\section{donde}

\section{$\mu_{w} \mathrm{Y} \mathcal{O}_{w}$}

son la media y desviación típica de la característica y k es la "distancia" entre los límites de control y la línea central, expresada en desviaciones estándares. Esta constante, usualmente tomará el valor 3. Cualquier valor obtenido que quede fuera de esos límites es indicativo de que el proceso objeto de estudio no está controlado. En nuestro caso se representa un Diagrama de Control de Atributos para controlar la fracción de disconformes $(\mathrm{w})$, por no medirse de forma cuantitativa lo que se está controlando (localización de la HC/alta hospitalaria o el proceso está bajo control y no localización de la HC/alta hospitalaria o el proceso está fuera de control), siendo la fracción de disconformes o de errores del Archivo de Historias Clínicas (\%):

$$
w=\frac{H C \text { No localizadas }+H C \text { con Incidencias }+H C \text { Prestadas }}{H C \text { Buscadas }} \cdot 100
$$

Al tratarse de muestras de tamaño variable, la variabilidad de cada una de ellas será distinta, lo cual implica el cálculo de límites de control específicos para cada una de las muestras (mes/año) según las fórmulas siguientes:

$$
\begin{gathered}
L S C=\bar{p}+3 \sqrt{\frac{\bar{p}(1-\bar{p})}{n_{i}}} \\
L C=\bar{p}=\frac{\sum_{i=1}^{m} x_{i}}{\sum_{i=1}^{m} n_{i}} \\
L I C=\bar{p}-3 \sqrt{\frac{\bar{p}(1-\bar{p})}{n_{i}}}
\end{gathered}
$$

Siendo $\mathrm{x}_{\mathrm{i}}$ el número de $\mathrm{HC}$ con errores en el mes $i, n_{i}$ el tamaño muestral de dicho mes y $\mathrm{k}=3$, donde la desviación estándar es estimada utilizando los datos de la muestra. Al ser la característica a medir un porcentaje, si aplicando esta formulación el LSC fuera mayor que 1 se asumirá que $\mathrm{LSC}=1$. Análogamente, si LIC es menor que 0 , tomaremos el valor 0

Con objeto de obtener una representación gráfica más fácil de interpretar, se considera interesante graficar los valores muestrales estandarizados.

$$
z_{i}=\frac{p_{i}-\bar{p}}{\sqrt{\frac{\bar{p}(1-\bar{p})}{n_{i}}}}
$$

En esta situación, la Línea Central y los Límites de Control serán:

$$
\mathrm{LSC}=+3, \mathrm{LC}=0, \mathrm{LIC}=-3
$$

Dado que la no existencia de errores supone un índice de calidad máximo, se excluye como regla de control la existencia de puntos por debajo del LIC, y se aplican además como reglas de control las rachas de puntos por debajo de $+1 /-1$ y +2/-2 sigmas, pues serán indicativas de cambios en la media del proceso, considerando pues las siguientes reglas: 
- Punto por encima de +3 sigmas.

- 2 puntos de los últimos 3 por encima de $+2 /-2$ sigmas.

- 4 puntos de los últimos 5 por encima de $+1 /-1$ sigmas.

- 8 puntos por encima/debajo de la línea central.

- 14 puntos seguidos alternándose.

La primera regla de control será indicativa de una fracción de disconformes demasiado alta en uno o varios periodos, teniendo en cuenta la media general de todo el proceso. Con las tres siguientes se pretende detectar cambios en la media del proceso y con la última, analizar la aleatoriedad del proceso.

El no cumplimiento de una o más de las reglas de control consideradas, permite concluir que el proceso está fuera de control, es decir, que el Archivo de Historias Clínicas no cumple criterios de calidad.

Posteriormente, se realizan Diagramas o Gráficos de Sumas Acumuladas (CUSUM), que permiten detectar con mayor claridad los cambios en la media del proceso de análisis. El CUSUM incorpora directamente toda la información, representando las sumas acumuladas de las desviaciones de los valores muestrales respecto de un valor objetivo, en nuestro caso el promedio de errores del archivo de HC (fracción media de disconformes) a lo largo de todo el período.

Suponiendo que el objetivo para la media del proceso es

$$
\bar{p}
$$

el gráfico de sumas acumuladas se formará representando la cantidad

$$
S A_{i}=\sum_{i=1}^{t}\left(p_{i}-\bar{p}\right)
$$

respecto al número de orden $(\mathrm{t})$ de la muestra. Si el proceso se mantiene bajo control en el objetivo

\section{$\bar{p}$}

la suma acumulada variará aleatoriamente respecto del valor cero. Sin embargo, si la media asciende a

$$
p_{1}>\bar{p}
$$

se apreciará una tendencia ascendente en la suma acumulada $\mathrm{SA}_{t}$. Por el contrario, si la media se desplaza a

$$
p_{2}<\bar{p}
$$

se apreciará una tendencia decreciente en $\mathrm{SA}_{\mathrm{t}}$. Por consiguiente, una tendencia determinada (positiva o negativa) se considerará como una evidencia de que la media del proceso se ha desplazado debido a la presencia de alguna causa, es decir, no debida al azar. Para establecer formalmente que el proceso está fuera de control se ha seguido un procedimiento numérico de tal forma que, en cada toma de muestra se calculan los 2 valores siguientes:

$$
S_{i}=\sum_{i=1}^{t}\left[p_{i}-(\bar{p}+F)\right] \quad T_{t}=\sum_{i=1}^{t}\left[p_{i}-(\bar{p}-F)\right]
$$

donde: $\mathrm{F}$ es un parámetro de la carta de control que normalmente vale $\mathrm{A}_{0} / 2$ siendo $\mathrm{A}_{0}$ el cambio que queremos detectar con prontitud y

$$
F=f \sigma_{w}
$$

considerando $f=0,5$ bajo el planteamiento de detectar cambios del orden de<smiles>[O-]</smiles>

Cuando algún valor $\mathrm{S}_{\mathrm{i}}$ ó $\mathrm{T}_{\mathrm{i}}$ cumple que $\mathrm{S}_{\mathrm{i}}>\mathrm{H}$ ó $\mathrm{T}_{\mathrm{i}}<-\mathrm{H}$

$$
\text { donde } H=h \sigma_{w} \text { y } \mathrm{h}=5
$$

el proceso se considera fuera de control. Si $\mathrm{S}_{\mathrm{i}}$ fuera negativo, se pone a 0 , de igual forma si $\mathrm{T}_{\mathrm{i}}$ fuera positivo, se pone a 0 . Una vez corregido el proceso los contadores $\mathrm{S}_{\mathrm{i}} \mathrm{y} \mathrm{T}_{\mathrm{i}}$ se pondrían a 0 . 


\section{RESULTADOS}

De las 33.032 altas hospitalarias buscadas durante los 421 días de la evaluación de la actividad del Archivo de Historias Clínicas se localizaron 31.443, lo que significa un Índice de Eficacia (IE) Global de 0,95 . El promedio de Historias Clínicas buscadas por día fue 78 y por mes 869 .

De las 16.702 Historias Clínicas que estaban en el Archivo 222 figuraban informáticamente fuera del mismo (figuraban prestadas) y 14.963 estaban fuera de él. No se localizaron $708(2,14 \%)$ y en 881 $(2,67 \%)$ estaba mal gestionado su registro informático, incluyendo los casos de Historias Clínicas incompletas y/o vacías (incidencias) que no debían de haber sido admitidas para su archivado. Siendo el porcentaje total de errores detectados del $4,81 \%$.

La distribución por años de los errores detectados en el Archivo de Historias Clínicas es la que se refleja en la figura 1, donde se observan porcentajes de errores superiores al $6 \%$ al inicio y final del período analizado. Correspondiendo al año 2006 el menor $\%$ de errores $(3,61 \%)$.

En la tabla 1 se muestran todos los resultados derivados de la evaluación de la calidad del Archivo de Historias Clínicas efectuada, a través de los Diagramas de Control de Shewhart. Así, en las columnas 3 y 4, aparecen los datos correspondientes al número de errores de archivado detectados en los diferentes momentos o muestras y el tamaño muestral (n). Las columnas 5 a 8 muestran la estadística asociada al Diagrama de Control con límites variables y, en las últimas columnas, esta misma estadística con los valores estandarizados, que constituye la base para la construcción del Diagrama de Control estandarizado. Destacando de dicha tabla el \% de errores obtenido en noviembre del 2008 con 55 errores de archivado (el 11,73\%), junio del 2008 con $14(10,45 \%)$, junio del 2007 con $20(9,09$ $\%)$, julio del 2005 con 147 (8,66\%), agosto de 2007 con $24(8,45 \%)$, diciembre del

Figura 1

Errores en el Archivo de Historias Clínicas

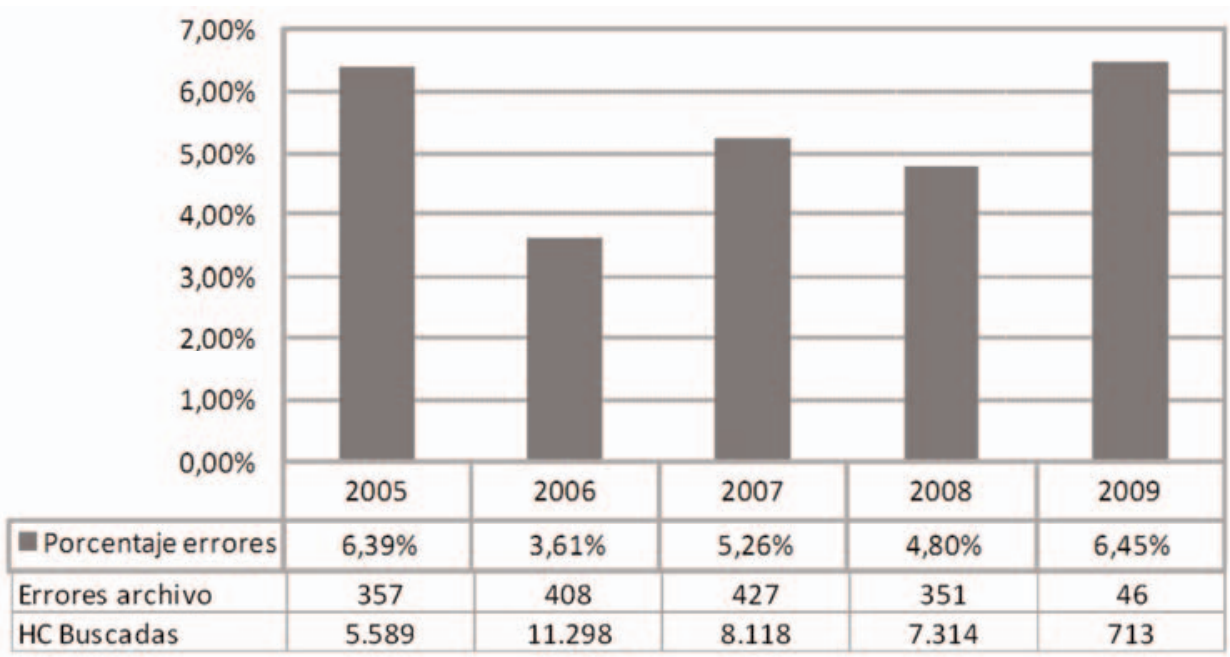


Tabla 1

Límites de control de los diagramas de Shewhart

\begin{tabular}{|c|c|c|c|c|c|c|c|c|c|c|c|}
\hline \multirow[b]{2}{*}{ Año } & \multirow[b]{2}{*}{ Mes } & \multirow[b]{2}{*}{ Errores } & \multirow[b]{2}{*}{$\mathrm{n}$} & \multicolumn{4}{|c|}{ Diagrama de control } & \multicolumn{4}{|c|}{ Diagrama de control estandarizado } \\
\hline & & & & $\begin{array}{c}\% \\
\text { Errores }\end{array}$ & LIC & LC & LSC & $\begin{array}{c}\% \text { errores } \\
\text { estandarizado }\end{array}$ & LIC & $\mathrm{LC}$ & LSC \\
\hline 2005 & Junio & 6 & 75 & $8,00 \%$ & $0,00 \%$ & $4,81 \%$ & $12,22 \%$ & 1,29 & -3 & 0 & 3 \\
\hline 2005 & Julio & 147 & 1.697 & $8,66 \%$ & $3,25 \%$ & $4,81 \%$ & $6,37 \%$ & 7,42 & -3 & 0 & 3 \\
\hline 2005 & Agosto & 2 & 62 & $3,23 \%$ & $0,00 \%$ & $4,81 \%$ & $12,96 \%$ & $-0,58$ & -3 & 0 & 3 \\
\hline 2005 & Octubre & 196 & 3.534 & $5,55 \%$ & $3,73 \%$ & $4,81 \%$ & $5,89 \%$ & 2,04 & -3 & 0 & 3 \\
\hline 2005 & Noviembre & 6 & 221 & $2,71 \%$ & $0,49 \%$ & $4,81 \%$ & $9,13 \%$ & $-1,46$ & -3 & 0 & 3 \\
\hline 2006 & Enero & 173 & 4.279 & $4,04 \%$ & $3,83 \%$ & $4,81 \%$ & $5,79 \%$ & $-2,35$ & -3 & 0 & 3 \\
\hline 2006 & Febrero & 3 & 305 & $0,98 \%$ & $1,13 \%$ & $4,81 \%$ & $8,49 \%$ & $-3,12$ & -3 & 0 & 3 \\
\hline 2006 & Marzo & 22 & 354 & $6,21 \%$ & $1,40 \%$ & $4,81 \%$ & $8,22 \%$ & 1,23 & -3 & 0 & 3 \\
\hline 2006 & Abril & 58 & 1.900 & $3,05 \%$ & $3,34 \%$ & $4,81 \%$ & $6,28 \%$ & $-3,58$ & -3 & 0 & 3 \\
\hline 2006 & Mayo & 4 & 284 & $1,41 \%$ & $1,00 \%$ & $4,81 \%$ & $8,62 \%$ & $-2,68$ & -3 & 0 & 3 \\
\hline 2006 & Junio & 11 & 282 & $3,90 \%$ & $0,99 \%$ & $4,81 \%$ & $8,63 \%$ & $-0,71$ & -3 & 0 & 3 \\
\hline 2006 & Julio & 57 & 1.463 & $3,90 \%$ & $3,13 \%$ & $4,81 \%$ & $6,49 \%$ & $-1,63$ & -3 & 0 & 3 \\
\hline 2006 & Septiembre & 4 & 362 & $1,10 \%$ & $1,44 \%$ & $4,81 \%$ & $8,18 \%$ & $-3,29$ & -3 & 0 & 3 \\
\hline 2006 & Octubre & 59 & 1.404 & $4,20 \%$ & $3,10 \%$ & $4,81 \%$ & $6,52 \%$ & $-1,06$ & -3 & 0 & 3 \\
\hline 2006 & Noviembre & 17 & 607 & $2,80 \%$ & $2,20 \%$ & $4,81 \%$ & $7,42 \%$ & $-2,31$ & -3 & 0 & 3 \\
\hline 2006 & Diciembre & 0 & 58 & $0,00 \%$ & $0,00 \%$ & $4,81 \%$ & $13,24 \%$ & $-1,71$ & -3 & 0 & 3 \\
\hline 2007 & Enero & 87 & 1.979 & $4,40 \%$ & $3,37 \%$ & $4,81 \%$ & $6,25 \%$ & $-0,86$ & -3 & 0 & 3 \\
\hline 2007 & Febrero & 40 & 500 & $8,00 \%$ & $1,94 \%$ & $4,81 \%$ & $7,68 \%$ & 3,33 & -3 & 0 & 3 \\
\hline 2007 & Marzo & 22 & 338 & $6,51 \%$ & $1,32 \%$ & $4,81 \%$ & $8,30 \%$ & 1,46 & -3 & 0 & 3 \\
\hline 2007 & Abril & 52 & 1.138 & $4,57 \%$ & $2,91 \%$ & $4,81 \%$ & $6,71 \%$ & $-0,38$ & -3 & 0 & 3 \\
\hline 2007 & Mayo & 40 & 569 & $7,03 \%$ & $2,12 \%$ & $4,81 \%$ & $7,50 \%$ & 2,47 & -3 & 0 & 3 \\
\hline 2007 & Junio & 20 & 220 & $9,09 \%$ & $0,48 \%$ & $4,81 \%$ & $9,14 \%$ & 2,97 & -3 & 0 & 3 \\
\hline 2007 & Julio & 72 & 1.469 & $4,90 \%$ & $3,14 \%$ & $4,81 \%$ & $6,49 \%$ & 0,16 & -3 & 0 & 3 \\
\hline 2007 & Agosto & 24 & 284 & $8,45 \%$ & $1,00 \%$ & $4,81 \%$ & $8,62 \%$ & 2,87 & -3 & 0 & 3 \\
\hline 2007 & Octubre & 52 & 1.254 & $4,15 \%$ & $3,00 \%$ & $4,81 \%$ & $6,62 \%$ & $-1,10$ & -3 & 0 & 3 \\
\hline 2007 & Noviembre & 18 & 367 & $4,90 \%$ & $1,46 \%$ & $4,81 \%$ & $8,16 \%$ & 0,08 & -3 & 0 & 3 \\
\hline 2008 & Enero & 61 & 1.129 & $5,40 \%$ & $2,90 \%$ & $4,81 \%$ & $6,72 \%$ & 0,93 & -3 & 0 & 3 \\
\hline 2008 & Febrero & 15 & 406 & $3,69 \%$ & $1,62 \%$ & $4,81 \%$ & $8,00 \%$ & $-1,05$ & -3 & 0 & 3 \\
\hline 2008 & Marzo & 3 & 38 & $7,89 \%$ & $0,00 \%$ & $4,81 \%$ & $15,22 \%$ & 0,89 & -3 & 0 & 3 \\
\hline 2008 & Abril & 34 & 1.322 & $2,57 \%$ & $3,04 \%$ & $4,81 \%$ & $6,58 \%$ & $-3,80$ & -3 & 0 & 3 \\
\hline 2008 & Mayo & 17 & 359 & $4,74 \%$ & $1,42 \%$ & $4,81 \%$ & $8,20 \%$ & $-0,07$ & -3 & 0 & 3 \\
\hline 2008 & Junio & 14 & 134 & $10,45 \%$ & $0,00 \%$ & $4,81 \%$ & $10,36 \%$ & 3,05 & -3 & 0 & 3 \\
\hline 2008 & Julio & 61 & 1.863 & $3,27 \%$ & $3,32 \%$ & $4,81 \%$ & $6,30 \%$ & $-3,10$ & -3 & 0 & 3 \\
\hline 2008 & Septiembre & 9 & 306 & $2,94 \%$ & $1,14 \%$ & $4,81 \%$ & $8,48 \%$ & $-1,53$ & -3 & 0 & 3 \\
\hline 2008 & Octubre & 62 & 1.038 & $5,97 \%$ & $2,82 \%$ & $4,81 \%$ & $6,80 \%$ & 1,75 & -3 & 0 & 3 \\
\hline 2008 & Noviembre & 55 & 469 & $11,73 \%$ & $1,85 \%$ & $4,81 \%$ & $7,77 \%$ & 7,00 & -3 & 0 & 3 \\
\hline 2008 & Diciembre & 20 & 250 & $8,00 \%$ & $0,75 \%$ & $4,81 \%$ & $8,87 \%$ & 2,36 & -3 & 0 & 3 \\
\hline 2009 & Enero & 46 & 713 & $6,45 \%$ & $2,41 \%$ & $4,81 \%$ & $7,21 \%$ & 2,05 & -3 & 0 & 3 \\
\hline
\end{tabular}


2008 con $20(8 \%)$, junio del 2005 con 6 $(8 \%)$, febrero del 2007 con $40(8 \%)$ y marzo del 2008 con 3 errores (el 7,89\%).

Con la representación gráfica de los correspondientes Diagramas de Control: Diagrama de Control con límites de control variable (figura 2) y Diagrama de Control estandarizado (figura 3) se ve como el proceso no está bajo control puesto que hay valores fuera de los Límites de Control. Destacando aquí los valores anormalmente altos, obtenidos tras la estandarización, en los meses de julio de $2005(7,42)$ y noviembre de $2008(7,00)$. También se sobrepasa el LSC en los meses correspondientes a febrero de $2007(3,33)$ y junio de 2008 $(3,05)$.

Se observó además un comportamiento no aleatorio de los valores alrededor de la línea central. En concreto, en el año 2006, a excepción del mes de marzo todos los valores se situaron por debajo de la línea central, mientras que durante 2007 tendieron a situarse por encima. Esto se traduce en 13 puntos que violan las reglas de control (tabla 2). Destacando por último la existencia de una alta variabilidad en los datos.

En la representación del Diagrama de Sumas Acumuladas (figura 4) se puede observar como la media del proceso ha ido variando a lo largo del tiempo, pudiendo delimitar 4 zonas temporales. En las dos primeras zonas, donde el gráfico muestra una tendencia descendente, se observa una continua mejora en la proporción de errores del Archivo de Historias Clínicas. Siendo los valores de las sumas acumuladas en la zona 1 mayores que 0 , lo que indica que en este tramo la calidad del Archivo fue menor que la media del proceso, mientras que en la zona 2 la calidad fue mayor, con valores menores que 0 . En las zonas 3 y 4 la calidad disminuye paulatinamente, mostrando así el gráfico una tendencia ascendente, man-

Figura 2

Diagrama de Control con límites de control variables

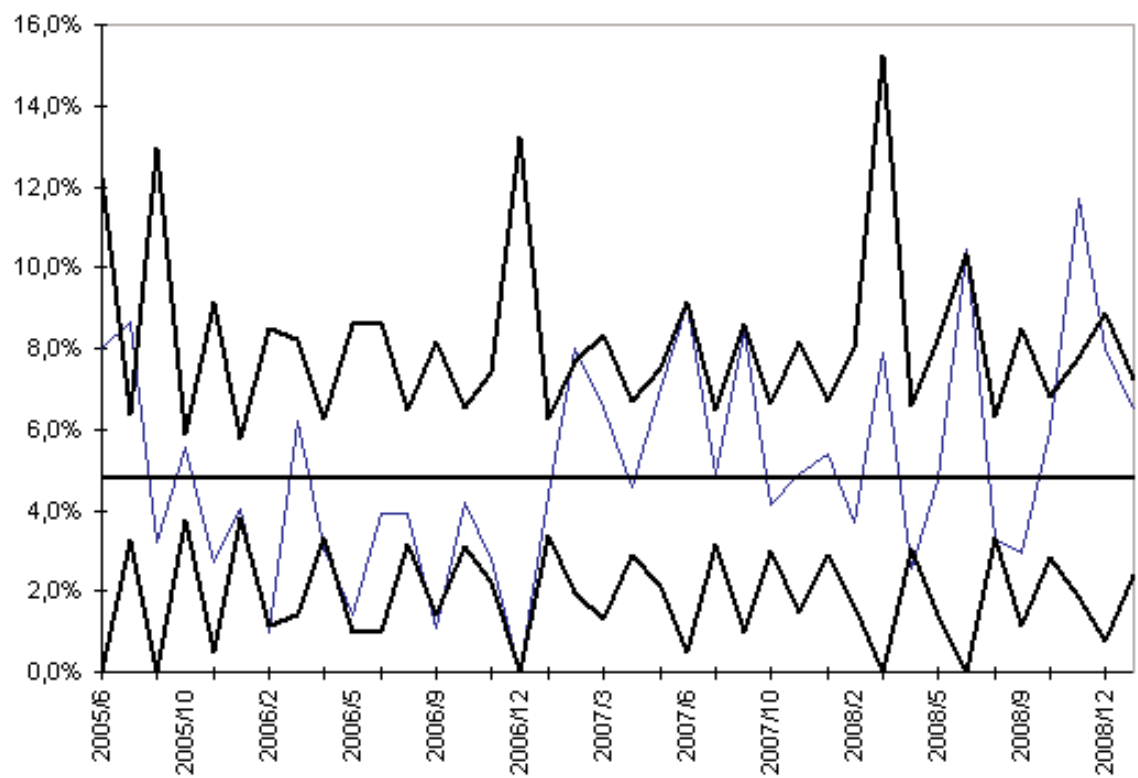

Año I Mex 
Figura 3

Diagrama de Control estandarizado

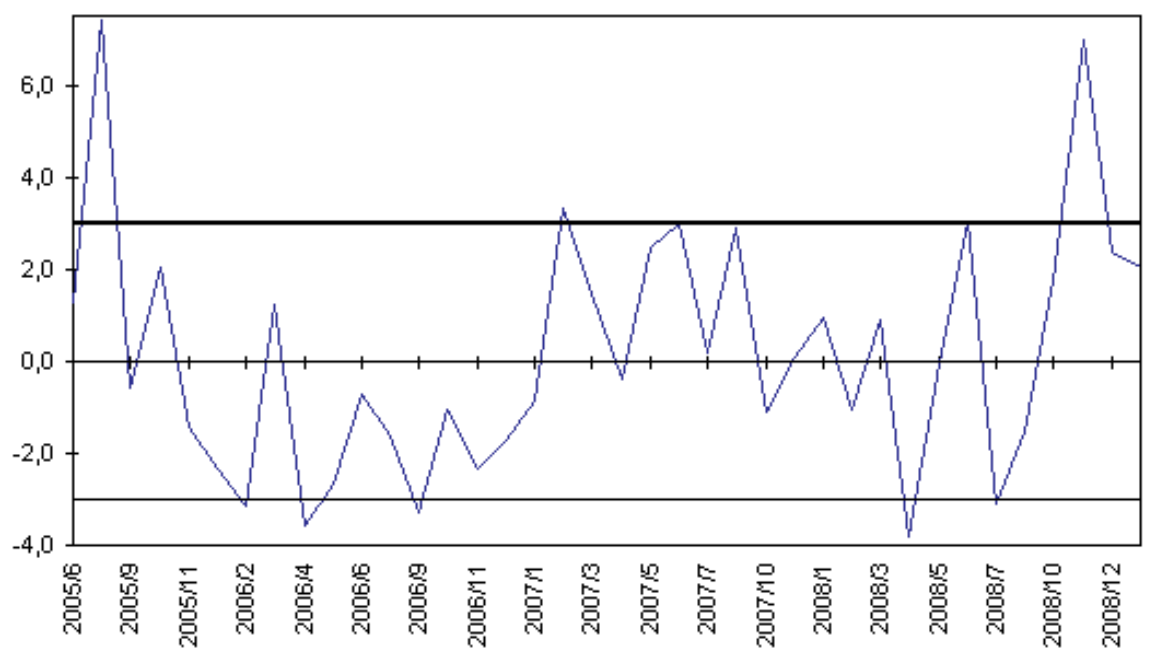

Año I Mes

Tabla 2

Violaciones de las reglas

\begin{tabular}{|l|l|}
\hline Año/Mes & Violaciones de puntos \\
\hline $2005 / 7$ & Mayor que +3 sigma \\
\hline $2005 / 10$ & 2 puntos de los últimos 3 por encima de +2 sigma \\
\hline $2006 / 2$ & 2 puntos de los últimos 3 por debajo de -2 sigma \\
\hline $2006 / 4$ & 2 puntos de los últimos 3 por debajo de -2 sigma \\
\hline $2006 / 4$ & 4 puntos de los últimos 5 por debajo de -1 sigma \\
\hline $2006 / 5$ & 2 puntos de los últimos 3 por debajo de 2 sigma \\
\hline $2006 / 5$ & 4 puntos de los últimos 5 por debajo de -1 sigma \\
\hline $2006 / 9$ & 4 puntos de los últimos 5 por debajo de -1 sigma \\
\hline $2006 / 10$ & 4 puntos de los últimos 5 por debajo de -1 sigma \\
\hline $2006 / 11$ & 2 puntos de los últimos 3 por debajo de -2 sigma \\
\hline $2006 / 11$ & 4 puntos de los últimos 5 por debajo de -1 sigma \\
\hline $2006 / 12$ & 4 puntos de los últimos 5 por debajo de -1 sigma \\
\hline $2006 / 12$ & 8 puntos consecutivos por debajo de la línea central \\
\hline $2007 / 1$ & 8 puntos consecutivos por debajo de la línea central \\
\hline $2007 / 2$ & Mayor que +3 sigma \\
\hline $2007 / 6$ & 2 puntos de los últimos 3 por encima de +2 sigma \\
\hline $2007 / 6$ & 4 puntos de los últimos 5 por encima de +1 sigma \\
\hline $2007 / 8$ & 2 puntos de los últimos 3 por encima de +2 sigma \\
\hline $2008 / 6$ & Mayor que +3 sigma \\
\hline $2008 / 11$ & Mayor que +3 sigma \\
\hline $2008 / 12$ & 2 puntos de los últimos 3 por encima de +2 sigma \\
\hline $2009 / 1$ & 2 puntos de los últimos 3 por encima de +2 sigma \\
\hline $2009 / 1$ & 4 puntos de los últimos 5 por encima de +1 sigma \\
\hline 17 puntos violan las reglas de control. \\
\hline
\end{tabular}


Tabla 3

Valores de los parámetros del test CUSUM

\begin{tabular}{|c|c|c|c|c|c|c|c|}
\hline Año & Mes & Errores & $\mathbf{n}$ & $\%$ Errores & $\mathbf{S A}_{\mathbf{t}}$ & $\mathrm{S}_{\mathrm{t}}$ & $\mathrm{T}_{\mathrm{t}}$ \\
\hline 2005 & Junio & 6 & 75 & $8,00 \%$ & $3,19 \%$ & $2,65 \%$ & $0,00 \%$ \\
\hline 2005 & Julio & 147 & 1697 & $8,66 \%$ & $7,04 \%$ & $5,95 \%$ & $0,00 \%$ \\
\hline 2005 & Agosto & 2 & 62 & $3,23 \%$ & $5,46 \%$ & $3,83 \%$ & $0,00 \%$ \\
\hline 2005 & Octubre & 196 & 3534 & $5,55 \%$ & $6,19 \%$ & $4,02 \%$ & $0,00 \%$ \\
\hline 2005 & Noviembre & 6 & 221 & $2,71 \%$ & $4,10 \%$ & $1,38 \%$ & $0,00 \%$ \\
\hline 2006 & Enero & 173 & 4279 & $4,04 \%$ & $3,33 \%$ & $0,07 \%$ & $0,00 \%$ \\
\hline 2006 & Febrero & 3 & 305 & $0,98 \%$ & $-0,50 \%$ & $0,00 \%$ & $-3,21 \%$ \\
\hline 2006 & Marzo & 22 & 354 & $6,21 \%$ & $0,91 \%$ & $0,86 \%$ & $0,00 \%$ \\
\hline 2006 & Abril & 58 & 1900 & $3,05 \%$ & $-0,85 \%$ & $0,00 \%$ & $-0,35 \%$ \\
\hline 2006 & Mayo & 4 & 284 & $1,41 \%$ & $-4,25 \%$ & $0,00 \%$ & $-2,86 \%$ \\
\hline 2006 & Junio & 11 & 282 & $3,90 \%$ & $-5,16 \%$ & $0,00 \%$ & $-0,37 \%$ \\
\hline 2006 & Julio & 57 & 1463 & $3,90 \%$ & $-6,08 \%$ & $0,00 \%$ & $-0,37 \%$ \\
\hline 2006 & Septiembre & 4 & 362 & $1,10 \%$ & $-9,78 \%$ & $0,00 \%$ & $-3,16 \%$ \\
\hline 2006 & Octubre & 59 & 1404 & $4,20 \%$ & $-10,39 \%$ & $0,00 \%$ & $-0,06 \%$ \\
\hline 2006 & Noviembre & 17 & 607 & $2,80 \%$ & $-12,40 \%$ & $0,00 \%$ & $-1,47 \%$ \\
\hline 2006 & Diciembre & 0 & 58 & $0,00 \%$ & $-17,21 \%$ & $0,00 \%$ & $-4,27 \%$ \\
\hline 2007 & Enero & 87 & 1979 & $4,40 \%$ & $-17,63 \%$ & $0,00 \%$ & $0,00 \%$ \\
\hline 2007 & Febrero & 40 & 500 & $8,00 \%$ & $-14,44 \%$ & $2,65 \%$ & $0,00 \%$ \\
\hline 2007 & Marzo & 22 & 338 & $6,51 \%$ & $-12,74 \%$ & $3,80 \%$ & $0,00 \%$ \\
\hline 2007 & Abril & 52 & 1138 & $4,57 \%$ & $-12,98 \%$ & $3,02 \%$ & $0,00 \%$ \\
\hline 2007 & Mayo & 40 & 569 & $7,03 \%$ & $-10,76 \%$ & $4,69 \%$ & $0,00 \%$ \\
\hline 2007 & Junio & 20 & 220 & $9,09 \%$ & $-6,48 \%$ & $8,43 \%$ & $0,00 \%$ \\
\hline 2007 & Julio & 72 & 1469 & $4,90 \%$ & $-6,39 \%$ & $7,98 \%$ & $0,00 \%$ \\
\hline 2007 & Agosto & 24 & 284 & $8,45 \%$ & $-2,75 \%$ & $11,07 \%$ & $0,00 \%$ \\
\hline 2007 & Octubre & 52 & 1254 & $4,15 \%$ & $-3,41 \%$ & $9,87 \%$ & $0,00 \%$ \\
\hline 2007 & Noviembre & 18 & 367 & $4,90 \%$ & $-3,32 \%$ & $9,42 \%$ & $0,00 \%$ \\
\hline 2008 & Enero & 61 & 1129 & $5,40 \%$ & $-2,73 \%$ & $9,47 \%$ & $0,00 \%$ \\
\hline 2008 & Febrero & 15 & 406 & $3,69 \%$ & $-3,84 \%$ & $7,81 \%$ & $0,00 \%$ \\
\hline 2008 & Marzo & 3 & 38 & $7,89 \%$ & $-0,76 \%$ & $10,35 \%$ & $0,00 \%$ \\
\hline 2008 & Abril & 34 & 1322 & $2,57 \%$ & $-3,00 \%$ & $7,57 \%$ & $0,00 \%$ \\
\hline 2008 & Mayo & 17 & 359 & $4,74 \%$ & $-3,07 \%$ & $6,95 \%$ & $0,00 \%$ \\
\hline 2008 & Junio & 14 & 134 & $10,45 \%$ & $2,57 \%$ & $12,04 \%$ & $0,00 \%$ \\
\hline 2008 & Julio & 61 & 1863 & $3,27 \%$ & $1,03 \%$ & $9,96 \%$ & $0,00 \%$ \\
\hline 2008 & Septiembre & 9 & 306 & $2,94 \%$ & $-0,84 \%$ & $7,55 \%$ & $0,00 \%$ \\
\hline 2008 & Octubre & 62 & 1038 & $5,97 \%$ & $0,32 \%$ & $8,17 \%$ & $0,00 \%$ \\
\hline 2008 & Noviembre & 55 & 469 & $11,73 \%$ & $7,24 \%$ & $14,54 \%$ & $0,00 \%$ \\
\hline 2008 & Diciembre & 20 & 250 & $8,00 \%$ & $10,43 \%$ & $17,19 \%$ & $0,00 \%$ \\
\hline 2009 & Enero & 46 & 713 & $6,45 \%$ & $12,07 \%$ & $18,28 \%$ & $0,00 \%$ \\
\hline
\end{tabular}


Figura 4

Sumas acumuladas porcentaje de errores

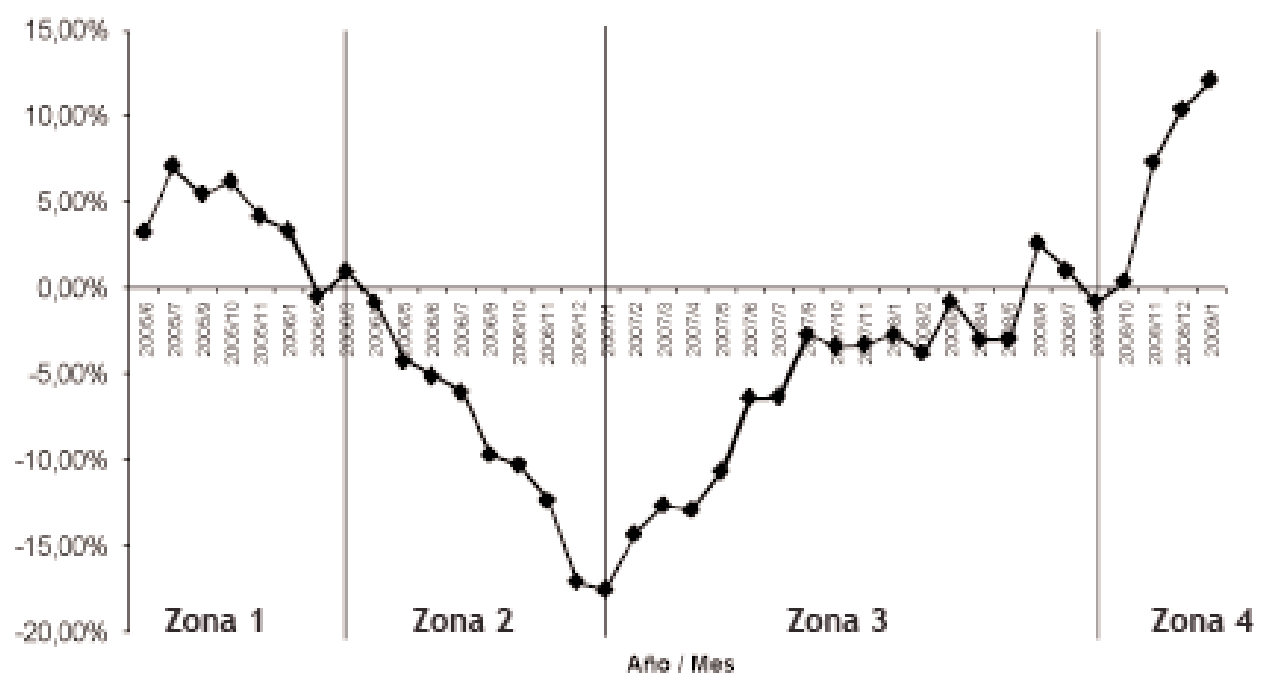

Figura 5

Diagrama CUSUM porcentaje de errores

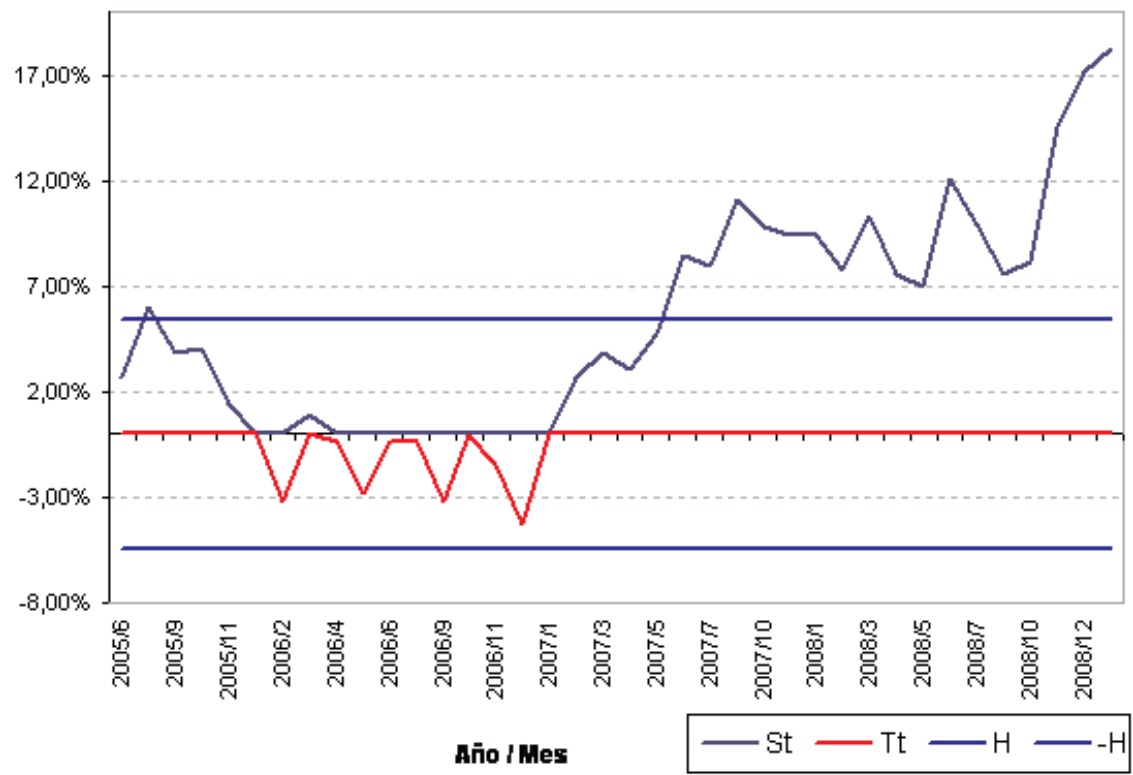


teniéndose por lo general su valor menor que la media del proceso en la zona 3 (valor de suma acumulada $<0$ ) para pasar a ser mayor (valor suma acumulada $>0$ ) en la zona 4.

En la tabla 3 se muestran los cálculos del Diagrama CUSUM. En la sexta columna figuran las sumas acumuladas representadas en la figura 4, y las dos últimas columnas corresponden al test destinado a evaluar los cambios significativos en la media del proceso, donde los Límites de Control serán $H=5,43$ para la serie $S_{t} y-H=-5,43$ para $T_{t}$, suponiendo, tal y como se expone en el apartado material y método $\mathrm{h}=5 \mathrm{y} \mathrm{f}=0,5$.

$\mathrm{Y}$ al aplicar el test CUSUM en su representación gráfica (figura 5), se observó un punto fuera de control al inicio de la serie $(5,95)$, concretamente en julio de 2005 , si bien hasta junio de 2007 la media se situó ligeramente por debajo de la media global $(4,70 \%$ frente a $4,81 \%)$, valor considerado como objetivo (target) del test realizado. A partir de julio de 2007 se apreció un cambio estadísticamente significativo en la media $(5,02 \%)$, dado que todos los puntos de la serie $\mathrm{S}_{\mathrm{t}}$ están por encima del límite de control, siendo más acusado este efecto al final del periodo.

\section{DISCUSIÓN}

La necesidad de tener que acceder al total de las altas hospitalarias, y en un plazo límite de tiempo, hace que sea necesaria una monitorización de dicha actividad, para ver las posibles desviaciones ante las posibles interferencias que puedan existir a lo largo del tiempo y que nos acercan o alejan de los objetivos de calidad establecidos. Tal y como se muestra en las representaciones gráficas de las sumas acumuladas se puede observar cómo existen dos tendencias claras a lo largo del periodo de estudio en cuanto a la calidad de la actividad del Archivo de Historias Clínicas, una primera de clara mejora progresiva de la calidad y otra de un empeoramiento progresivo siendo mayor al final del período, lo que se puede deber primero, y entre otras cosas, a una mayor estabilidad del personal, con la consiguiente formación y adiestramiento, y a la existencia de una situación laboral conocida sin grandes cambios (no cambios drásticos en metodología e infraestructuras); y segundo, el mayor empeoramiento coincide con el traslado a un nuevo centro, dónde el Archivo pasaba a ser ya de $\mathrm{HC}$ digitalizada, para el Área de Hospitalización e informatizado para el Área de Consultas Externas. Existiendo un tiempo dónde la incorporación de nuevo personal, ajeno incluso a la institución, además de la implantación de nuevas tecnologías, con lo que ello conlleva ${ }^{12,13}$, y el mantenimiento de la actividad habitual del centro, que hacía necesario la coexistencia de dos soportes diferentes de la documentación clínica, digitalizado y en papel, hacía difícil el desarrollar un programa de control de calidad dentro de su actividad regular ${ }^{14}$. Incluso la generalización de la informatización del informe de alta, no supuso una mejora de la calidad del Archivo de Historias Clínicas. Aunque con ello la Unidad de Codificación Clínica ya disponía de una fuente de información para su labor, codificándose entonces sólo lo reflejado en él, y no lo existente en todo el episodio asistencial como era lo habitual.

Por todo lo dicho, se podría intuir que la media de calidad del proceso varía a lo largo del periodo considerado, si bien, tal y como se ha explicado anteriormente, los Gráficos de Shewhart no son los más adecuados para analizar esto, pudiéndose dar el caso en que un proceso esté bajo control y sin embargo la media del proceso experimente cambios estadísticamente significativos.

La adecuación del método de sumas acumuladas (CUSUM) para el control de calidad de un Archivo de Historias Clínicas lo determina el hecho de que estas representaciones, como ya se ha demostrado 
en múltiples aplicaciones incluso dentro del campo de la Medicina ${ }^{19-25}$, nos permiten detectar cambios referidos a alejamientos de un estándar de calidad que no son posible evidenciar con el tratamiento en bloque de los datos; pudiendo así investigar las posibles causas, analizarlas y establecer medidas correctoras, tanto de forma retrospectiva como prospectiva $^{19-20,24-25}$, ya que se trata de conseguir que la calidad del Archivo de Historias Clínicas, a través de la monitorización continua del cumplimento de unos estándares de calidad, se mantenga constante a lo largo del tiempo.

Hay que decir que en el estudio se ha considerado como error o fallo tanto el no localizar la $\mathrm{HC}$, que ésta tenga incidencias o que esté mal gestionada informáticamente, sin hacer distinción del tipo concreto de error detectado. Esto que puede suponer el inconveniente de la no ponderación en función del tipo de error, atribuyendo así una importancia diferente a cada uno, puede ser objeto de posteriores estudios.

Como recomendación se puede decir que desde una unidad externa al Archivo de Historias Clínicas, como es la Unidad de Codificación Clínica, y a través de la incorporación de técnicas de control estadístico de calidad, como las utilizadas en este trabajo, de fácil implantación y que ya se han mostrado eficaces para monitorizar diversas actividades en otros servicios o unidades clínicas ${ }^{19-23}$, se puede llevar a cabo un control continuo de la calidad de la actividad del Archivo de Historias Clínicas. La incorporación de la nuevas TIC's en los centros asistenciales, y por consiguiente en los diversos servicios o unidades clínicas ${ }^{12-13}$, y en nuestro caso concreto la implantación de la $\mathrm{HC}$ electrónica, que supondrá el manejo electrónico de los datos, hará que mejoren los resultados de los diversos indicadores de calidad, alcanzando o a lo sumo aproximándonos más a los estándares preestablecidos, pero ello no eliminará la necesidad del desarrollo de programas de control de calidad cara a la obtención de un proceso de calidad, en definitiva a la consecución de una asistencia sanitaria e investigación de calidad. Ya que no hay que olvidar los diferentes aspectos o atributos de la calidad.

Como conclusión podemos decir que la calidad del Archivo de Historias Clínicas no se mantiene constante a lo largo del tiempo, siendo ésta una cualidad deseable en cualquier proceso. La evaluación continua o monitorización según unos estándares de calidad predeterminados con técnicas estadísticas de control de calidad como las utilizadas en este estudio hace que podamos detectar los momentos a partir de los cuales el proceso se desvía de esos estándares, es decir, que no está bajo control, permitiéndonos así el análisis y la identificación de los posibles factores que contribuyen a dicha desviación y la consiguiente implantación de medidas para su corrección.

\section{BIBLIOGRAFÍA}

1. Secretaria General para el Sistema Nacional de Salud. Resolución 1/92 sobre el establecimiento de un CMBD al alta hospitalaria. Madrid: Ministerio de Sanidad y Consumo; 1992.

2. Boletín Oficial de Castilla y León. Decreto 28/2007, de 15 de marzo, por el que se establece el Sistema de Información de Enfermedades Asistidas, se regula el Conjunto Mínimo Básico de Datos (CMBD) al alta hospitalaria y procedimientos ambulatorios especializados y se crea el Registro del CMBD de la Comunidad de Castilla y León. BOCyL núm 57 de 21/03/2007.

3. Dirección General de Planificación, Calidad, Ordenación y Formación. Manual de Procedimiento del Conjunto Mínimo Básico de Datos. Valladolid: Consejería de Sanidad. Junta de Castilla y León; 2008.

4. López Domínguez O. El Servicio de Admisión y Documentación Clínica. En: Gestión de pacientes en el hospital. Madrid: Olalla ediciones; 1997. p. 85-134.

5. Moreno Vernis M. El archivo de historias clínicas. En: Gestión de pacientes en el hospital. López Domínguez O. Madrid: Olalla ediciones; 1997. p. 253337. 
6. Tejero Álvarez M. Documentación Clínica y Archivo. Madrid: Ediciones Díaz de Santos SA; 2004.

7. Joint Commission on Accreditation of Healthcare Organizations. Estándares internacionales de acreditación de Hospitales. Barcelona: Joint Commission International Accreditation Fundación Avedis Donabedian; 2001.

8. AENOR. UNE-EN ISO 9001:2000. Sistemas de gestión de la Calidad. Requisitos. Madrid: Asociación Española para la Normalización y la Certificación; 2001.

9. European Foundation for Quality Management. European Excellence Model. Brussels 2000. Disponible en : http//www.efqm.org.

10. Tejedor Fernández M, Aljama Alcántara M, Delgado Jiménez C, Morilla Aceijas F, Paz León U, Ruiz Tárraga R. Programa de calidad en un archivo de historias clínicas. Papeles Médicos 2001; 11(3): 107-112.

11.Yetano J, López Arbeloa P. La calidad y el servicio de documentación clínica. Med Clin (Barc). 1993; 101:301-2.

12. Ramos López JM, Cuchí Alfaro M, Sánchez Molano MA. Archivo de historias clínicas Digitalizado, solución previa a la Historia Clínica Electrónica. Papeles Médicos 2009; 18 (2): 4-10.

13. Häyrinen K, Saranto K, Nykänen P. Definition, structure, content, use and impacts of electronic health records: a review of the research literature. Int J Med Inform. 2008 May; 77(5): 291-304. Epub 207 Oct 22.

14. Lorenzoni L, Da Cas R, Aparo UL. The quality of abstracting medical information from the medical record: The impact of training programmes. Int J Qual Health Care. 1999 Jun; 11(3):209-13.

15. Ishikawa K. Guía de Control de Calidad. New Cork: UNIPUB; 1985.

16. Peña D, Prat A. Cómo controlar la calidad. Manuales IMPL. Madrid: Ministerio de Industria y Energía; 1986.

17. British Standards Institution. Guide to data analysis and quality control using cusum techniques. Uses and value of cusum charts in business, industry, commerce and public service. London: BSI; 2003.

18. Williams SM, Parry BR, Schlup MMT. Quality control: an application of the cusum. BMJ 1992; 304(6838): 1359-60.
19. Baptista Macarroff WM, Castroman Espasandín P. Utilización del método de suma acumulada (cusum) para la evaluación continua de la calidad de la analgesia en una Unidad de Dolor Agudo Postoperatorio. Rev Esp Anestesiol Reanim. 2007; 54 (1): 11-6.

20. Morton AP, Whitby M, McLaws ML, Dobson A, McElwain S, Looke D et al. The application of statistical process control charts to the detection and monitoring of hospital-acquired infections. J Qual Clin Pract. 2001 Dec; 21(4):112-7.

21. Barrington KJ, Denson-Lino J, Bloch R, Finer NN. Sequential analysis for control in the neonatal intensive care unit. J Pediatr. 2001 Dec; 139(6): 778-84.

22. Grigg OA, Farewell VT, Spiegelhalter DJ. Use of risk-adjusted CUSUM and RSTRT charts for monitoring in medical contexts. Stat Methods Med Res. 2003 Mar; 12(2):147-70.

23. Ioli P, Rauek S, Santamarina R, Gonorazky S. Cómo mejorar el control de los riesgos perioperatorios en endarterectomía carotídea. Aplicación del Método de Suma Acumulativa (CUSUM). Rev Neurol Arg. 2007; 32(1):35-9.

24. Noyez L. Control charts, Cusum techniques and funnel plots. A review of methods for monitoring performance in healthcare. Interact Cardiovasc Thorac Surg. 2009; 9: 494-9.

25. Biau DJ, Porcher R. A method for monitoring a process from an out of control to an in control state: Application to the learning curve. Stat Med. 2010 Aug $15 ; 29$ (18):1900-9. 